\title{
Effect of antimicrobial exposure on AcrAB expression in Salmonella enterica subspecies enterica serovar Choleraesuis
}

\author{
Masaru Usui ${ }^{1,2}$, Hidetaka Nagai ${ }^{1}$, Mototaka Hiki ${ }^{1}$, Yutaka Tamura ${ }^{2}$ and Tetsuo Asai ${ }^{1}$ * \\ ${ }^{1}$ National Veterinary Assay Laboratory, Ministry of Agriculture, Forestry and Fisheries, Kokubunji, Tokyo, Japan \\ 2 School of Veterinary Medicine, Rakuno Gakuen University, Ebetsu, Hokkaido, Japan
}

Edited by:

Kunihiko Nishino, Osaka University, Japan

Reviewed by:

Julie Zilles, University of Illinois

Urbana Champaign, USA

Glenn Kaatz, John D. Dingell VA

Medical Center, USA

\section{*Correspondence:}

Tetsuo Asai, National Veterinary Assay Laboratory, Ministry of Agriculture,

Forestry and Fisheries, 1-15-1 Tokura Kokubunji, Tokyo 185-8511, Japan.

e-mail: asai-t@nval.maff.go.jp
Understanding the impact of antimicrobial use on the emergence of resistant bacteria is imperative to prevent its emergence. For instance, activation of the AcrAB efflux pumps is responsible for the emergence of antimicrobial-resistant Salmonella strains. Here, we examined the expression levels of acrB and its multiple regulator genes (RamA, SoxS, MarA, and Rob) in 17 field isolates of $S$. Choleraesuis by using quantitative PCR methods. The expression of $a c r B$ increased in eight of the field isolates $(P<0.05)$. The expression of $a c r B$ was associated with that of ramA in one isolate, soxS in one isolate, and both these genes in six isolates. Thereafter, to examine the effect of selected antimicrobials (enrofloxacin, ampicillin, oxytetracycline, kanamycin, and spectinomycin) on the expression of $a c r B$ and its regulator genes, mutants derived from five isolates of $S$. Choleraesuis were selected by culture on antimicrobial-containing plates. The expression of $a c r B$ and ramA was higher in the mutants selected using enrofloxacin (3.3-6.3- and 24.5-37.7-fold, respectively), ampicillin (1.8-7.7- and 16.1-55.9-fold, respectively), oxytetracycline (1.7-3.3and 3.2-31.1-fold, respectively), and kanamycin (1.6-2.2- and 5.6-26.4-fold, respectively), which are AcrAB substrates, than in each of the parental strains $(P<0.05)$. In contrast, in AcrAB substrate-selected mutants, the expression of soxS, marA, and rob remained similar to that in parental strains. Of the four antimicrobials, the level of ramA expression was significantly higher in the enrofloxacin- and ampicillin-selected mutants than in the oxytetracycline- and kanamycin-selected mutants $(P<0.05)$, whereas the expression levels of $a c r B$ and multiple regulator genes in spectinomycin-selected mutants were similar to those in each parental strain. These data suggest that exposure to antimicrobials that are $A c r A B$ substrates enhance the activation of the AcrAB efflux pump via RamA, but not via SoxS, MarA, or Rob in S. Choleraesuis.

Keywords: AcrAB efflux pump, antimicrobial resistance, RamA, Salmonella Choleraesuis, SoxS

\section{INTRODUCTION}

Salmonella enterica subspecies enterica serovar Choleraesuis is a bacterial pathogen that causes severe diarrhea, pneumonia, and septicemia in pigs and in elderly and immunocompromised humans (Chiu et al., 2004). These bacteria sometimes cause severe infections, necessitating antimicrobial treatment of affected patients. A high rate of multidrug resistance among $S$. Choleraesuis has been reported in several countries (Lee et al., 2009; Asai et al., 2010), raising a concern for public and animal health.

Activation of an efflux system that removes the drug from the cell is one antimicrobial resistance mechanism in bacteria (Chen et al., 2007; Li and Nikaido, 2009). The AcrAB-TolC system is the main multidrug efflux system in Gram-negative bacteria (Giraud et al., 2000). The expression level of acrAB mRNAs correlates with efflux activities and is regulated by global regulatory proteins and local repressors (Rosenberg et al., 2003; Li and Nikaido, 2004; Olliver et al., 2004; Abouzeed et al., 2008). In particular, the expression of $a c r A B$ is regulated by the global regulators, RamA, SoxS, MarA, and Rob (Rosenberg et al., 2003; Abouzeed et al.,
2008). Moreover, mutations within the local repressor AcrR contribute to the overexpression of acrAB (Olliver et al., 2004). In $S$. Typhimurium and $S$. Haardt, RamA mainly regulates the expression of acrAB (Figure 1; Nikaido et al., 2008; Zheng et al., 2009; Kim and Woo, 2011). Other regulators, SoxS and MarA, are also known to regulate $a c r A B$ expression, but the contributions of SoxS and MarA to antimicrobial susceptibilities are lower than that of RamA (Abouzeed et al., 2008). Little is known about Rob and its contribution to the enhancement of AcrAB in Salmonella (Rosenberg et al., 2003). In S. Pullorum, the expression of acrB, which shifts from sensitivity to resistance against bile salt (deoxycholate, an $A c r A B$ substrate), was independent of ramA, soxS, marA, and rob expression (Usui et al., 2011).

We have previously recorded the various efflux activity levels of $S$. Choleraesuis under field conditions, suggesting that elevated efflux activities are related to the emergence of fluoroquinolone resistance (Usui et al., 2009). In fluoroquinoloneselected $S$. Choleraesuis, efflux pumps including AcrAB were enhanced and resulted in decreasing susceptibilities to several 
antimicrobials (Usui et al., 2010). However, little is known about roles of regulator genes for efflux pumps in $S$. Choleraesuis. In this study, we examined the expression levels of $a c r A B$ and multiple regulator genes in 17 clinical swine isolates of $S$. Choleraesuis

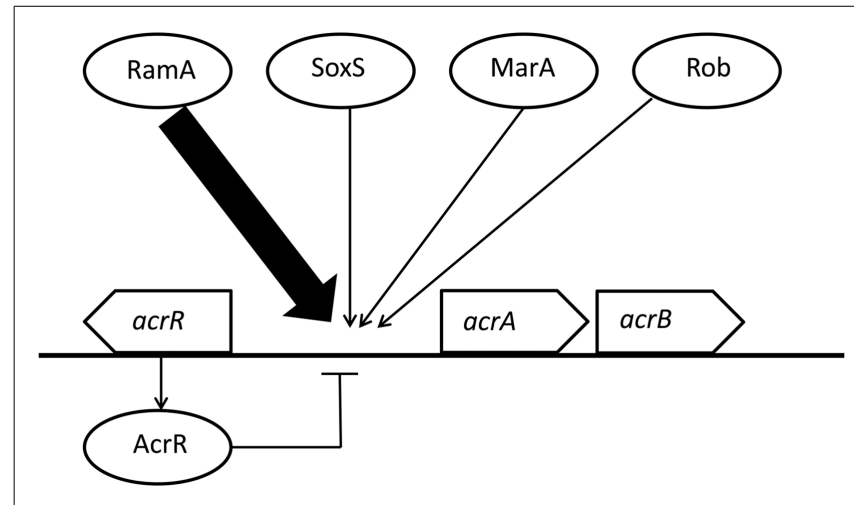

FIGURE 1 | Regulation of acrAB expression by multiple regulators in Salmonella Typhimurium and $\boldsymbol{S}$. Haardt. This figure was produced using data from the literature (see main text). Functional interactions are represented as arrows for activation/induction and as " $\dashv$ " for repression. The size of arrows indicates the estimated impact. and antimicrobial-selected mutants in order to understand the impact of antimicrobial exposure on the efflux activities in this organism.

\section{MATERIALS AND METHODS BACTERIAL STRAINS}

Seventeen strains of $S$. Choleraesuis, obtained from different diseased pigs between 2001 and 2005 (Asai et al., 2010), were used in this study (Table 1). These strains comprised 6 nalidixic acid-resistant strains (low enrofloxacinaccumulation), and 11 nalidixic acid-susceptible strains (two strains: low enrofloxacin-accumulation; three strains: intermediate enrofloxacin-accumulation; six strains: high enrofloxacinaccumulation) as previously recorded (Usui et al., 2009). The intracellular concentration of enrofloxacin was significantly lower in nalidixic acid-resistant isolates, and the nalidixic acidsusceptible isolates ZSC-8 and 582 as compared to other susceptible isolates with the exception of isolates 143, ZSC-12, and 1002 $(P<0.1$; Usui et al., 2009). The intracellular enrofloxacin concentrations of isolates 143, ZSC-12, and 1002 were at an intermediate level. Minimum inhibitory concentrations (MIC) of enrofloxacin, ampicillin, oxytetracycline, and kanamycin had been determined in our previous study (Asai et al., 2010). MIC of spectinomycin was

Table 1 | Characterization of Salmonella Choleraesuis used in this study.

\begin{tabular}{|c|c|c|c|c|c|c|c|c|}
\hline \multirow[t]{2}{*}{ Strain no. } & \multicolumn{2}{|c|}{ Susceptibility to nalidixic acid ${ }^{a}$} & \multicolumn{2}{|c|}{ Enrofloxacin-accumulation } & \multicolumn{2}{|c|}{ mRNA expression } & \multicolumn{2}{|c|}{ Mutations } \\
\hline & Category $^{\mathbf{b}}$ & $\operatorname{MIC}(\mathbf{m g} / \mathrm{L})^{b}$ & Categoryc & $\left(\mathrm{ng} / 10^{5} \mathrm{cfu}\right)^{\mathrm{c}}$ & $\operatorname{acr} A^{\mathrm{d}}$ & $a c r B^{d}$ & $\operatorname{AcrR}^{\mathbf{e}}$ & Binding site \\
\hline 13-PLS-6 & Resistance & 256 & Low & 8.2 & $1.6 \pm 0.2^{*}$ & $1.8 \pm 0.2^{*}$ & WT & WT \\
\hline 14-PLS-21 & Resistance & $>512$ & Low & 8.7 & $3.9 \pm 0.3^{*}$ & $2.7 \pm 0.2^{*}$ & WT & WT \\
\hline 16-PLS-45 & Resistance & $>512$ & Low & 6.5 & $1.5 \pm 0.1^{*}$ & $1.9 \pm 0.3^{*}$ & Q78ter & WT \\
\hline 16-PLS-46 & Resistance & $>512$ & Low & 6.6 & $2.4 \pm 0.2^{*}$ & $2.4 \pm 0.4^{*}$ & Q78ter & WT \\
\hline sal-1372 & Resistance & $>512$ & Low & 6.3 & $1.8 \pm 0.1^{*}$ & $2.2 \pm 0.2^{*}$ & WT & WT \\
\hline 16-PLS-33 & Resistance & $>512$ & Low & 5.1 & $1.5 \pm 0.1^{*}$ & $1.9 \pm 0.2^{*}$ & Q78ter & WT \\
\hline ZSC-8 & Susceptible & 8 & Low & 8.1 & $1.7 \pm 0.1^{*}$ & $1.8 \pm 0.2^{*}$ & Q78ter & WT \\
\hline 582 & Susceptible & 8 & Low & 7.2 & $1.4 \pm 0.1^{*}$ & $1.4 \pm 0.2^{*}$ & Q78ter & WT \\
\hline 143 & Susceptible & 8 & Intermediate & 9.1 & $1.1 \pm 0.1$ & $1.3 \pm 0.1$ & Q78ter & WT \\
\hline ZSC-12 & Susceptible & 4 & Intermediate & 9.5 & $1.0 \pm 0.1$ & $1.0 \pm 0.1$ & Q78ter & WT \\
\hline 1002 & Susceptible & 4 & Intermediate & 9.8 & $1.1 \pm 0.2$ & $1.2 \pm 0.2$ & Q78ter & WT \\
\hline 916 & Susceptible & 4 & High & 10.7 & $1.1 \pm 0.1$ & $1.1 \pm 0.1$ & Q78ter & WT \\
\hline 03-197-1 & Susceptible & 2 & High & 11.4 & $0.9 \pm 0.1$ & $0.8 \pm 0.1$ & Q78ter & WT \\
\hline 03-228-1 & Susceptible & 2 & High & 10.7 & $1.0 \pm 0.2$ & $1.1 \pm 0.1$ & Q78ter & WT \\
\hline ZSC-14-1 & Susceptible & 4 & High & 11.0 & $0.9 \pm 0.1$ & $1.1 \pm 0.1$ & Q78ter & WT \\
\hline ZSC-40 & Susceptible & 4 & High & 10.5 & $0.9 \pm 0.2$ & $0.9 \pm 0.2$ & WT & WT \\
\hline 419 & Susceptible & 4 & High & 12.7 & $1.0 \pm 0.2$ & $0.9 \pm 0.1$ & Q78ter & WT \\
\hline
\end{tabular}

MIC, minimum inhibitory concentration; WT, wild-type sequence.

${ }^{a} \mathrm{MIC}$ break point of nalidixic acid is $32 \mathrm{mg} / \mathrm{L}$.

${ }^{b}$ Data from a previous report (Asai et al., 2010).

${ }^{c}$ Data from a previous report (Usui et al., 2009).

${ }^{d}$ Relative expression level compared to the average for six high enrofloxacin-accumulating nalidixic acid-susceptible strains.

e Q78ter, stop codon mutation, glutamine-78-termination.

*Indicates mRNA expression is significantly higher than mRNA expression of six high enrofloxacin-accumulating nalidixic acid-susceptible strains (P < 0.05). 
determined by the broth microdilution method according to CLSI guidelines (Clinical and Laboratory Standard Institute, 2008).

\section{LABORATORY-SELECTED MUTANTS}

Five strains $(582,143,916,1002$, and 419), susceptible to all of the antimicrobial agents evaluated in this study, were selected as parent strains from which mutants were isolated. Cultures grown on agar containing various agents was employed, including three AcrAB substrates (ampicillin, oxytetracycline, and kanamycin) and one non-substrate (spectinomycin; Bailey et al., 2008; Ricci and Piddock, 2009a,b). For selection of mutants, $0.1 \mathrm{~mL}$ of each strain, adjusted to $10^{8}-10^{9} \mathrm{cfu} / \mathrm{mL}$ were exposed to $4 \times \mathrm{MIC}$ of the respective substance for each strain in the agar medium, as described previously (Ricci and Piddock, 2009b). A single strain was selected for each combination. In addition, as fluoroquinolone is an AcrAB substrates, enrofloxacin-selected mutants obtained in our previous report were used as control (Usui et al., 2010). MICs of antimicrobials for mutants were determined as described above.

\section{QUANTITATIVE PCR ANALYSIS OF EXPRESSION OF 16S rRNA, acrAB AND MULTIPLE GLOBAL REGULATORS}

To evaluate the genes conferring efflux pump activity and their multiple global regulators, quantitative PCR was applied to the detection and quantification of mRNAs (Usui et al., 2010, 2011). The preparation of cDNA was performed as described by Zheng et al. (2009), with slight modifications. In brief, extraction of total RNA from $5 \mathrm{~mL}$ of bacterial suspensions was carried out with an ISOGEN kit (Nippon Gene, Tokyo, Japan). cDNA was synthesized from these RNA samples using the PrimeScript RT reagent kit (TaKaRa, Shiga, Japan). Quantitative PCR was performed with SYBR premix EX TaqII (TaKaRa) on a One Step real-time system (Applied Biosystems, Foster City, CA, USA) according to the manufacturer's instructions. The oligonucleotide primers used for the detection of cDNA are listed in Table 2.
The yields of amplicons from acrA, acrB, $\operatorname{ram} A$, soxS, marA, and rob were normalized to those originating from 16S rRNA. Within bacterial cells, 16S rRNA was assumed to be transcribed at a constant rate throughout the growth conditions used in this study.

After normalization to the levels of 16S rRNA, gene expression was compared with the average values for the six high enrofloxacin-accumulating susceptible strains.

\section{acrR DNA SEQUENCE ANALYSIS}

Mutations in the local repressor gene, $a c r R$, and the regulatorbinding site were detected by direct DNA sequencing, using previously reported primer sets (Zheng et al., 2009). Amplification of the gene and purification of the resulting amplicon were performed as described before (Zheng et al., 2009). Nucleotide

Table 2 | Primers used in this study.

\begin{tabular}{lll}
\hline Primer name & Sequence $\left(\mathbf{5}^{\prime} \mathbf{-} \mathbf{3}^{\prime} \mathbf{)}\right.$ & Reference \\
\hline acrA-F & AAAACGGCAAAGCGAAGGT & Usui et al. (2011) \\
acrA-R & GTACCGGACTGCGGGAATT & Usui et al. (2011) \\
acrB-rt1 & GGCATTGGGTATGACTGGAC & Zheng et al. (2009) \\
acrB-rt2 & GCATTACGGAGAACGGGATAG & Zheng et al. (2009) \\
ramA-rt1 & TTTCCGCTCAGGTTATCGAC & Zheng et al. (2009) \\
ramA-rt2 & CGGGCAATATCATCAATACG & Zheng et al. (2009) \\
soxS-rt1 & AAATCGGGCTACTCCAAG & Zheng et al. (2009) \\
soxS-rt2 & TACTCGCCTAATGTTTGATG & Zheng et al. (2009) \\
marA-rt1 & ATTCTCTATCTGGCGGAAC & Zheng et al. (2009) \\
marA-rt2 & CGGGTCAATGTTTGCTGTG & Zheng et al. (2009) \\
robA-rt1 & TATTCCGCCAGTGCTTTATG & Zheng et al. (2009) \\
robA-rt2 & CCTGCTCATCGTCTTTCTCC & Zheng et al. (2009) \\
16S rRNA-F & CCAGCAGCCGCGGTAAT & Usui et al. (2010) \\
16S rRNA-R & TTTACGCCCAGTAATTCCGATT & Usui et al. (2010) \\
& & \\
\hline
\end{tabular}

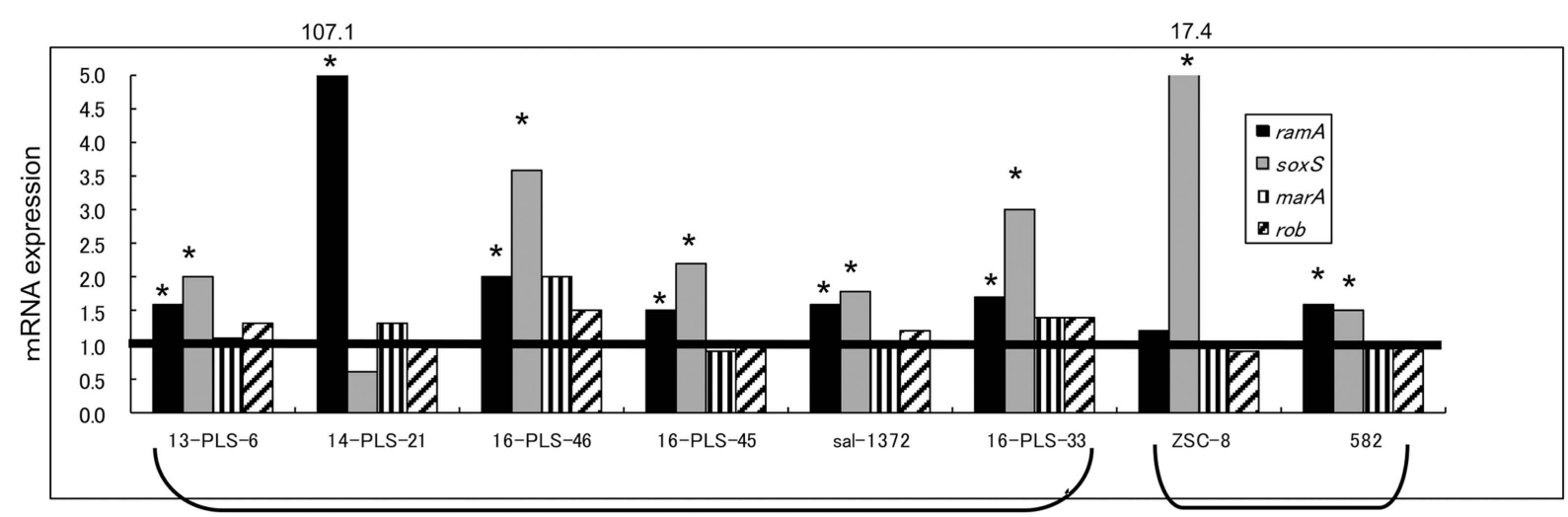

Nalidixic acid-resistant strains

Low enrofloxacin-accumulation nalidixic acid-susceptible strains

FIGURE 2 | Expression of multiple regulator genes in field isolates of $\boldsymbol{S}$. Choleraesuis. The expression of multiple regulator genes in resistant strains and low enrofloxacin-accumulating nalidixic acid-susceptible strains was compared to the average expression of the corresponding genes in high enrofloxacin-accumulating nalidixic acid-susceptible strains ( $n=6)$. Horizontal line indicates the average gene expression in the six susceptible strains, which expressions were set to be 1.0. ${ }^{*}$ Indicates that mRNA expression is significantly higher in a strain compared to mRNA expression in the six susceptible strains $(P<0.05)$. 
Table 3 | The MICs of several antimicrobials and gene expressions of acrB and multiple regulator genes in laboratory-selected mutants.

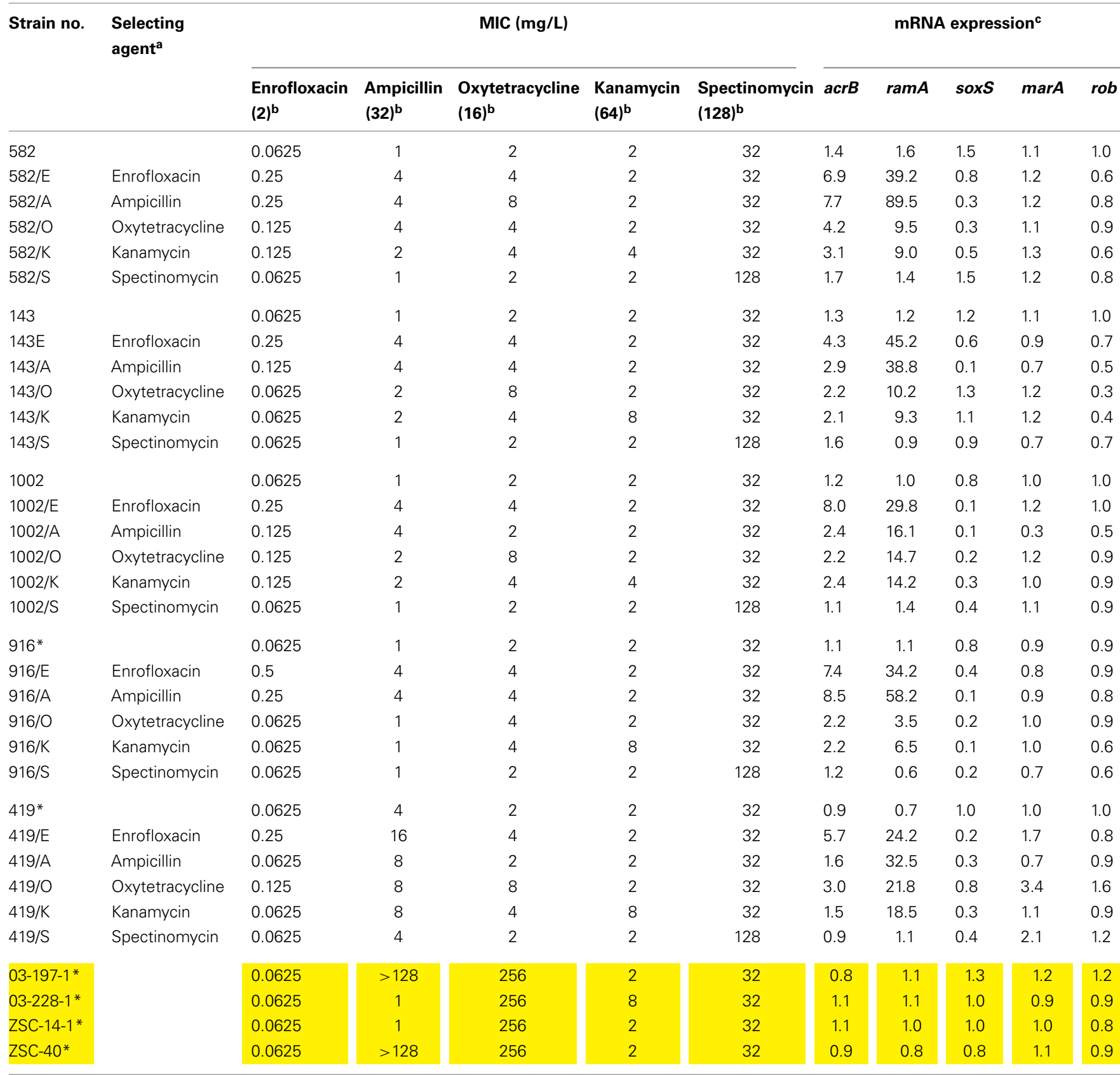

MIC, minimum inhibitory concentration.

${ }^{a}$ Mutants were selected by exposing to fourth the MIC of the antimicrobials for each susceptible strain.

${ }^{b} \mathrm{MIC}$ data of antimicrobials except for spectinomycin in parental strains from a previous report (Asai et al., 2010). Parenthesis indicates break points.

${ }^{c}$ Relative expression level of acrB and multiple regulator genes compared to average of six high accumulation susceptible strains represented as asterisks.

sequences were determined using a BigDye Terminator v3.1 Cycle Sequencing Kit (Applied Biosystems) with a 3130 Genetic Analyzer (Applied Biosystems).

\section{STATISTICAL ANALYSIS}

Student's $t$-test was used to compare results between and within experiments. $P$-values $<0.05$ were considered significant.

\section{RESULTS}

RELATIONSHIP BETWEEN THE ENHANCEMENT OF EFFLUX PUMPS AND THE EXPRESSION OF acrAB

Expression of acrA and $a c r B$ in the six resistant strains and in the two low enrofloxacin-accumulation nalidixic acid-susceptible strains was significantly higher than that in the other susceptible strains $(P<0.05$; Table 1). Expression levels of acr $B$ correlated 
significantly with those of acrA in this study (correlation coefficient: 0.89 ).

\section{RELATIONSHIP BETWEEN INCREASED EXPRESSION OF acrB AND GLOBAL REGULATORS}

Of the eight strains with a high $a c r B$ expression level, six strains showed higher levels of both $\operatorname{ram} A$ and soxS expression compared with the average values for the six high accumulating susceptible strains $(P<0.05$; Figure 2$)$. The remaining strains showed a higher level of ramA or soxS expression $(P<0.05)$ : the $14-$ PLS-21 strain showed only a higher expression level of $\operatorname{ram} A$ (107.1-fold \pm 42.0 -fold), while the ZSC-8 strain showed only a higher expression level of soxS (17.4-fold \pm 2.7 -fold). Neither the expression of marA nor that of rob changed in any of the strains tested.

\section{SEOUENCING OF THE LOCAL REPRESSOR GENE acrR AND OF THE REGULATOR-BINDING SITE}

A stop codon mutation in AcrR (glutamine-78-to-terminal; Q78ter) was found in 13 strains (Table 1). No significant relationship was found between the Q78ter stop codon mutation in AcrR and the expression of $a c r B$. None of the strains tested had a point mutation in the regulator-binding site, which affects the $a \operatorname{cr} B$ expression, previously determined using the $S$. serovar Typhimurium strain BN18 (Olliver et al., 2004).

\section{GENE EXPRESSION IN LABORATORY-SELECTED MUTANTS}

Expression levels of both $a c r B$ and $\operatorname{ram} A$ increased significantly in AcrAB substrate-selected mutants compared to these levels in parental strains $(P<0.05$; Table 3). Among four AcrAB substrates, a higher level of ramA expression was observed in the enrofloxacinand ampicillin-selected mutants than in the oxytetracycline- and kanamycin-selected mutants $(P<0.05)$. In contrast, the expression of soxS decreased significantly in AcrAB substrate-selected mutants compared to that in parental strains $(P<0.05$; Table 3$)$. MICs of AcrAB substrates were increased in AcrAB substrateselected mutants as compared with those in the parental strains (Table 3).

Expression of $a c r B$ and multiple regulator genes remained stable in spectinomycin-selected mutants. In spectinomycin-selected mutants, MICs of AcrAB substrates were of the same level as compared with those in the parental strains (Table 3 ).

\section{DISCUSSION}

The current study showed that the expression of $\operatorname{ram} A$ and/or soxS was associated with $\operatorname{acr} A B$ overexpression in field isolates of $S$. Choleraesuis. In this study, expression of $\operatorname{ram} A$, but not soxS, marA, or rob, was enhanced in laboratory-derived $S$. Choleraesuis mutants selected using antimicrobials that are AcrAB substrates. Several previous studies have reported that

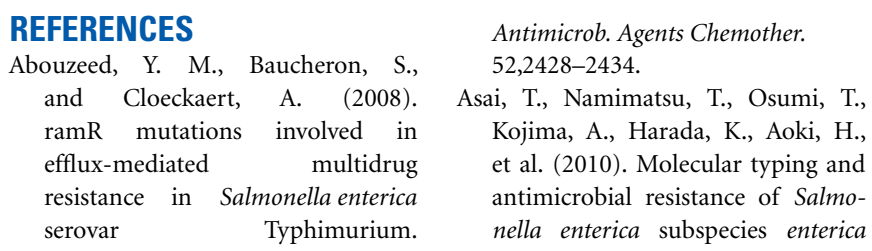

$\operatorname{ram} A$, but not soxS, is involved in activation of the AcrABTolC system in S. Typhimurium (Ricci et al., 2006; Bailey et al., 2008; Zheng et al., 2009). Interestingly, in this study, soxS expression was downregulated in AcrAB substrate-selected mutants of $S$. Choleraesuis. In addition, enrofloxacin- and ampicillinselected mutants derived from the ZSC- 8 strain with a high level of soxS expression, showed increased expression of $\operatorname{ramA}$, but not of soxS (data not shown). Several studies have suggested that increased expression of ramA downregulates soxS expression (Nikaido et al., 2008; O'Regan et al., 2009). These results suggest that the $a c r A$ and $a c r B$ expression in antimicrobialselected mutants of $S$. Choleraesuis were dependent on ram $A$ expression.

The present study showed that increased levels of both ramA and soxS expression were demonstrated by some field isolates of $S$. Choleraesuis upon activation of the AcrAB-TolC system. There was a marked difference in soxS expression between the field isolates and the laboratory-derived strains. As all of the strains used in this study were isolated from diseased pigs, these isolates would have been exposed to antimicrobials and disinfectants used in disease treatment and hygiene management. This suggests that the resistance in some field isolates was mediated through a different mechanism, and therefore could have arisen from a different selective pressure.

Activation of the AcrAB efflux pump is responsible for the emergence of fluoroquinolone-resistant Salmonella strains (Ricci et al., 2006; Usui et al., 2009). In Japan, the description of veterinary fluoroquinolone drugs includes an explanation that the drug is considered as a second-line drug. In the AcrAB substrateselected mutants, the level of $a c r B$ expression generally increased, depending on ramA expression. It is possible that the use of an AcrAB substrate as a first-line drug crucially selects strains of $S$. Choleraesuis with activated AcrAB efflux systems. Among AcrAB substrates used in this study, the degree of $a c r B$ expression may be different due to exposure to the various antimicrobial agents. Therefore, the antimicrobial class used as first-line drugs may be associated with the frequency of fluoroquinolone resistance in bacterial strains. Further studies are needed in order to clarify the effects of each antimicrobial on $a c r B$ expression in these bacteria.

In conclusion, the AcrAB-TolC efflux system in field isolates of $S$. Choleraesuis is likely regulated by external factors in the environment, such as antimicrobials and drug residues via ramA and soxS. In particular, exposure of AcrAB substrates enhances activation of AcrAB via RamA in S. Choleraesuis.

\section{ACKNOWLEDGMENTS}

We would like to thank the staff at the Prefectural Livestock Hygiene Service Center for providing S. Choleraesuis isolates. This work was supported in part by a Grant-in Aid from the Ministry of Health, Labor and Welfare of Japan (H21-Shokuhin-Ippan-013).

serovar Choleraesuis isolates from diseased pigs in Japan. Comp. Immunol. Microbiol. Infect. Dis. 33, 109-119.

Bailey, A. M., Paulsen, I. T., and Piddock, L. J. (2008). RamA confers multidrug resistance in
Salmonella enterica via increased expression of $a c r B$, which is inhibited by chlorpromazine. Antimicrob. Agents Chemother. 52, 3604-3611.

Chen, S., Cui, S., McDermott, P. F., Zhao, S., White, D. G., Paulsen, I., et al. (2007). Contribution of target gene 
mutations and efflux to decreased susceptibility of Salmonella enterica serovar Typhimurium to fluoroquinolones and other antimicrobials. Antimicrob. Agents Chemother. 51, 535-542.

Chiu, C. H., Su, L. H., and Chu, C. (2004). Salmonella enterica serotype Choleraesuis: epidemiology, pathogenesis, clinical disease, and treatment. Clin. Microbiol. Rev. 17, 311-322.

Clinical and Laboratory Standard Institute. (2008). Performance Standards for Antimicrobial Disk and Dilution Susceptibility Tests for Bacteria Isolated from Animals, Third Edition: Approved Standard M31-A3. Wayne, PA: Clinical and Laboratory Standard Institute.

Giraud, E., Cloeckaert, A., Kerboeuf, D., and Chaslus-Dancla, E. (2000). Evidence for active efflux as the primary mechanism of resistance to ciprofloxacin in Salmonella enterica serovar Typhimurium. Antimicrob. Agents Chemother. 44, 1223-1228.

Kim, K. Y., and Woo, G. J. (2011). Expression of $a c r B$ and $\operatorname{ram} A$ in fluoroquinolone resistant mutants from multi-drug resistant Salmonella enterica serovar Haardt. Lett. Appl. Microbiol. 52, 484-490.

Lee, H. Y., Su, L. H., Tsai, M. H., Kim, S. W., Chang, H. H., Jung, S. I., et al. (2009). High rate of reduced susceptibility to ciprofloxacin and ceftriaxone among nontyphoid Salmonella clinical isolates in Asia. Antimicrob. Agents Chemother. 53, 2696-2699.
Li, X. Z., and Nikaido, H. (2004). Effluxmediated drug resistance in bacteria. Drugs 64, 159-204.

Li, X. Z., and Nikaido, H. (2009). Efflux-mediated drug resistance in bacteria: an update. Drugs 69, 1555-1623.

Nikaido, E., Yamaguchi, A., and Nishino, K. (2008). AcrAB multidrug efflux pump regulation in Salmonella enterica serovar Typhimurium by RamA in response to environmental signals. J. Biol. Chem. 283, 24245-24253.

Olliver, A., Valle, M., Chaslus-Dancla, E., and Cloeckaert, A. (2004). Role of an acrR mutation in multidrug resistance of in vitroselected fluoroquinolone-resistant mutants of Salmonella enterica serovar Typhimurium. FEMS Microbiol. Lett. 238, 267-272.

O’Regan, E., Quinn, T., Pages, J. M., McCusker, M., Piddock, L., and Fanning, S. (2009). Multiple regulatory pathways associated with high-level ciprofloxacin and multidrug resistance in Salmonella enterica serovar enteritidis: involvement of RamA and other global regulators. Antimicrob. Agents Chemother. 53, 1080-1087.

Ricci, V., and Piddock, L. J. (2009a). Ciprofloxacin selects for multidrug resistance in Salmonella enterica serovar Typhimurium mediated by at least two different pathways. $J$. Antimicrob. Chemother. 63, 909-916.

Ricci, V., and Piddock, L. J. (2009b). Only for substrate antibiotics are a functional AcrAB-TolC efflux pump and RamA required to select multidrug-resistant Salmonella Typhimurium. J. Antimicrob. Chemother. 64, 654-657.

Ricci, V., Tzakas, P., Buckley, A., and Piddock, L. J. (2006). Ciprofloxacinresistant Salmonella enterica serovar Typhimurium strains are difficult to select in the absence of AcrB and TolC. Antimicrob. Agents Chemother. 50, 38-42.

Rosenberg, E. Y., Bertenthal, D., Nilles, M. L., Bertrand, K. P., and Nikaido, H. (2003). Bile salts and fatty acids induce the expression of Escherichia coli AcrAB multidrug efflux pump through their interaction with Rob regulatory protein. Mol. Microbiol. $48,1609-1619$.

Usui, M., Asai, T., and Sato, S. (2011). Low expression of $a c r B$ in the deoxycholate-sensitive strains of Salmonella enterica subspecies enterica serovar Pullorum. Microbiol. Immunol. 55, 366-368.

Usui, M., Uchiyama, M., Baba, K., Nagai, H., Yamamoto, Y., and Asai, T. (2010). Contribution of enhanced efflux to reduced susceptibility of Salmonella enterica serovar Choleraesuis to fluoroquinolone and other antimicrobials. J. Vet. Med. Sci. 73 , 279-282.

Usui, M., Uchiyama, M., Iwanaka, M., Nagai, H., Yamamoto, Y., and Asai, T. (2009). Intracellular concentrations of enrofloxacin in quinoloneresistant Salmonella enterica subspecies enterica serovar Choleraesuis. Int. J. Antimicrob. Agents 34 592-595.
Zheng, J., Cui, S., and Meng, J. (2009). Effect of transcriptional activators RamA and SoxS on expression of multidrug efflux pumps AcrAB and AcrEF in fluoroquinoloneresistant Salmonella Typhimurium. J. Antimicrob. Chemother. 63 95-102.

Conflict of Interest Statement: The authors declare that the research was conducted in the absence of any commercial or financial relationships that could be construed as a potential conflict of interest.

Received: 26 November 2012; accepted: 25 February 2013; published online: 14 March 2013.

Citation: Usui M, Nagai H, Hiki M, Tamura $Y$ and Asai $T$ (2013) Effect of antimicrobial exposure on AcrAB expression in Salmonella enterica subspecies enterica serovar Choleraesuis. Front. Microbiol. 4:53. doi 10.3389/fmicb.2013.00053

This article was submitted to Frontiers in Antimicrobials, Resistance and Chemotherapy, a specialty of Frontiers in Microbiology.

Copyright (0) 2013 Usui, Nagai, Hiki, Tamura and Asai. This is an openaccess article distributed under the terms of the Creative Commons Attribution License, which permits use, distribution and reproduction in other forums, provided the original authors and source are credited and subject to any copyright notices concerning any third-party graphics etc. 